Ann. Biol. anim. Bioch. Biophys., I974, 14 (I), II7-I29.

\title{
ÉVOLUTION DES DIFFÉRENTS TYPES DE DÉPÔTS ADIPEUX APRÈS LA NAISSANCE CHEZ DES AGNEAUX MERINOS D'ARLES NORMAUX OU HYPOPHYSECTOMISÉS
}

\author{
A. VÉZINHET, M. PRUD'HON et M. BÉNÉVENT \\ avec la collaboration technique de E. Bouthier et A. Benhacine \\ Station de Physiologie animale, \\ École nationale supérieure agronomique, I. N. R. A., \\ Place Viala, \\ 34060 Montpellier Cedex

\section{RÉSUMÉ}

L'évolution des différents types de dépôts adipeux pendant la vie postnatale a été étudiée chez 99 agneaux Mérinos d'Arles normaux des deux sexes, et chez 45 agneaux ayant subi une hypophysectomie ou un simulacre d'opération. Certains des agneaux hypophysectomisés recevaient un traitement chronique à l'hormone somatotrope bovine (BGH). L'ensemble des dépôts adipeux prend, par rapport au poids vide, une importance relative croissante au cours de la vie postnatale pendant la période étudiée. Au niveau des différents types de dépôts on note une proportion à peu près constante du gras de ratis ( 10 à $\mathbf{~} 2$ p. Ioo du gras total) et des dépôts intermusculaires ( 25 à $30 \mathrm{p}$. Ioo du gras total). Les dépôts périrénaux et pelviens perdent de l'importance tandis que les sous-cutanés et la toilette en gagnent. Cette dernière évolue de façon analogue aux dépôts totaux. Une forte variabilité affecte les données obtenues, elle est surtout marquée dans le cas des gras périrénal et sous-cutanés.

L'hypophysectomie induit une adiposité accrue des carcasses. La répartition des différents dépôts adipeux des agneaux hypophysectomisés diffère assez sensiblement de celle des agneaux normaux du même âge, mais diffère peu de celle observée chez les agneaux sacrifiés à l'âge auquel a été pratiqué l'ablation hypophysaire, à l'exception du tissu périrénal, plus développé chez les agneaux opérés.

Le traitement chronique à la $\mathrm{BGH}$ des hypophysectomisés entraîne un amaigrissement des carcasses confirmant ainsi le rôle lipolytique de cette hormone.

\section{INTRODUC'TION}

L'excès de dépôts adipeux, tout en grevant le prix de revient, est généralement une cause de dépréciation des carcasses, bien qu'un état d'engraissement optimum soit un important facteur de qualité. Mais ce dernier dépend autant de la répartition 
des différents dépôts que de leur masse totale, alors que de nombreux travaux, dont FLAMAN'T et BocCARD (I966) ont fait la revue, se sont efforcés d'apprécier, à l'aide de différents critères, l'adiposité globale des carcasses d'ovins.

En fait, comme l'ont souligné SHAFriR et WERTHEIMER (I965), rares sont les études concernant la localisation des dépôts adipeux et l'évolution de leur importance relative au cours de la croissance, ou sous l'influence de facteurs internes comme les hormones sexuelles, l'insuline, les corticoïdes, etc. On sait cependant qu'il existe des différences interspécifiques. Ainsi chez le Rat (REED et al., r930) et le Porc (RICHMOND et BERG, I97I ; MARTIN et al., I972; HENRY, I972) l'importance des dépôts adipeux sous-cutanés est prépondérante et peut atteindre plus des $2 / 3$ des dépôts adipeux totaux, alors que chez le Lapin l'importance de ces dépôts décroît avec l'âge et se fixe aux environs du tiers des dépôts totaux chez l'adulte (PRUD'HON, VEZINHET et CANTIER, I970). De même à l'intérieur d'une espèce la localisation des dépôts adipeux peut varier de façon notable. Chez les races ovines à grosse queue les dépôts adipeux de la région caudale peuvent prendre un développement considérable jusqu'à I2 p. IOO du poids vif selon KHAchadurian et al. (I966).

Cette localisation des dépôts adipeux et leur évolution sous l'effet de facteurs endocriniens, ont été assez peu étudiées. Dumont (I97I) soulignait que la topographie de la couverture graisseuse de la carcasse ovine et l'étude de ses facteurs de variation nous manquaient pour établir une méthode précise permettant de caractériser 1'état d'engraissement général. BOCCARD et DUPLAN (I96I) ont analysé l'importance respective des dépôts sous-cutanés et intermusculaires en fonction de la vitesse de croissance dans les différents morceaux de la découpe de carcasses d'un poids commercial. Russel, Doney et GunN (I97I) ont estimé, en pourcentages, la répartition des différents types de dépôts en fonction de l'état d'adiposité global de la carcasse. Mc CleLLAND et Russei, (I972) ont abordé l'étude de la distribution des différents dépôts chez deux races d'ovins. Dans notre laboratoire, BENEvent (I97I) a étudié l'allométrie par rapport au poids vif vide, des différents types de gras chez des agneaux Mérinos d'Arles des deux sexes ayant une même croissance moyenne. PRUd'hON, REYNE et GaRAmbors (I972) ont souligné l'intérêt d'utiliser les dépôts adipeux du petit épiploon ou " toilette " pour prédire l'importance des dépôts totaux tout au long de la croissance. Enfin, VÉzINHET (I973) a considéré l'influence de l'hypophysectomie et de traitements chroniques à l'hormone somatotrope sur le gras total dissécable.

Dans cette étude nous nous proposons de décrire l'évolution de l'importance relative des différents dépôts par rapport au gras total, au cours de la croissance d'agneaux Mérinos d'Arles, et les conséquences sur cette répartition de l'ablation de l'hypophyse suivie ou non d'injections d'hormone somatotrope bovine.

\section{MATÉRIEL ET MÉTHODES}

Un effectif de 54 mâles et 45 femelles Mérinos d'Arles, issus de l'élevage du laboratoire, a permis de réaliser cette étude sur des animaux normaux. Ils étaient répartis de $\mathrm{I}$ à $25^{\circ}$ jours suivant les âges, les effectifs et les poids figurant au tableau I. Certains de ces agneaux servaient également de témoins à 22 mâles et 23 femelles ayant subi une hypophysectomie ou un simulacre d'opération à 25,50 ou 100 jours et respectivement abattus à 100, 150 et 250 jours, après avoir 
reçu ou non un traitement chronique à l'hormone somatotrope bovine (BGH). Les conditions expérimentales et le détail des groupes expérimentaux figurent dans l'étude de V́́zINHET (I973). Nous rappellerons cependant que l'hypophysectomie était pratiquée par la voie parapharyngienne selon Denamur et Martinet (non publié), que le traitement chronique à l'hormone BGH était réalisé à raison de 3 injections intramusculaires par semaine à la dose de $3 \mathrm{mg}$ par $\mathrm{kg}$ de poids vif. Des dissections d'animaux normaux ayant été effectuées au début et à la fin de chaque période éudiée, les valeurs obtenues ont permis d'avoir une image de l'évolution normale des tissus adipeux et d'estimer l'influence de l'hypophysectomie, du traitement à la BGH et du choc opératoire chez les sujets qui ont subi un simulacre d'opération et que nous nommerons pseudoopérés. Après l'abattage nous avons procédé à l'enlèvement des viscères et notamment des dépôts adipeux mésentériques, périrénaux et pelviens. La carcasse était séparée en deux suivant un plan médiosagittal. Une demi carcasse était ensuite entièrement disséquée suivant la méthode décrite par BÉnḱvENT (I968) ou une méthode simplifiée (Prud'hon, Reyne, Garambors, I972). Les dépôts adipeux internes, intermusculaires et sous-cutanés étaient prélevés et pesés par catégorie au cours de ces opérations.

\section{RÉSULTATS}

\section{I. - Agneaux normaux}

L'évolution de la somme des dépôts adipeux par rapport au poids vif vide en fonction de l'âge est représentée sur la figure $I$, on notera :

- l'importance croissante des dépôts adipeux qui atteignent I3 p. Ioo environ chez les mâles et I 8 p. I00 chez les femelles âgées de $25^{\circ}$ jours ;

- 1'existence d'un palier dans le développement relatif du tissu adipeux des mâles et des femelles entre 50 et roo jours ;

- la supériorité constante à partir de 25 jours de l'adiposité des femelles par rapport aux mâles.

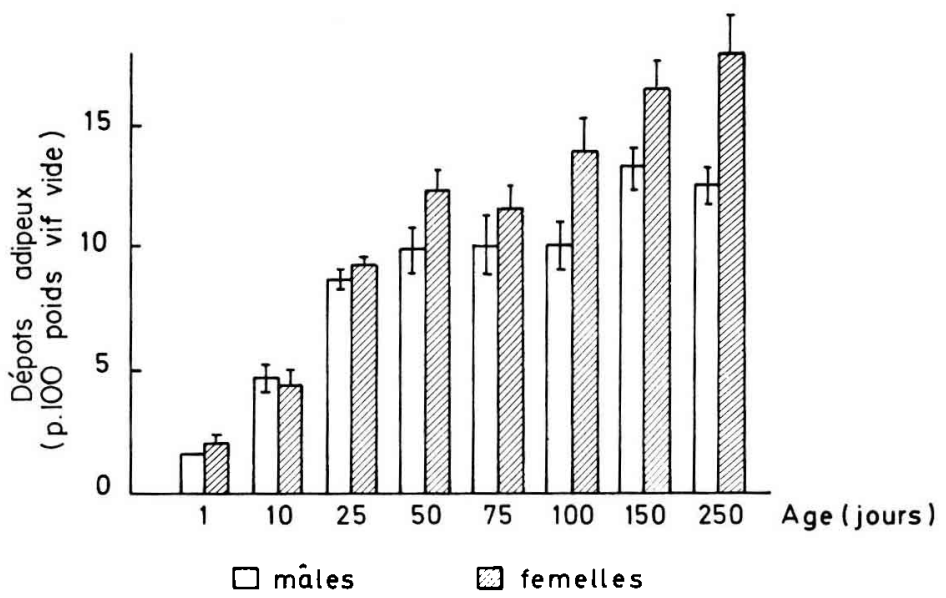

Fra. I. - Evolution en fonction de l'âge de la somme (moyenne \pm erreur standard) des dépôts adipeux en p. 100 du poids vif vide pour des agneaux Mérinos d'Arles des deux sexes

La figure 2 représente en pourcentage du gras total dissécable, les évolutions des différents types de dépôts.

- Le gras du petit épiploon ou de " toilette " évolue dans les deux sexes de façon semblable à la somme des dépôts adipeux comme cela a été montré par BÉNÉvENT (I97I) et PRUd'hon et al. (I972). 
- Le gras du grand épiploon ou de "ratis " conserve un taux relativement constant tout au long de la croissance et dans les deux sexes, si l'on excepte le stade de naissance notamment chez les mâles (où compte tenu des faibles dépôts une légère différence entraîne des modifications importantes de pourcentage).
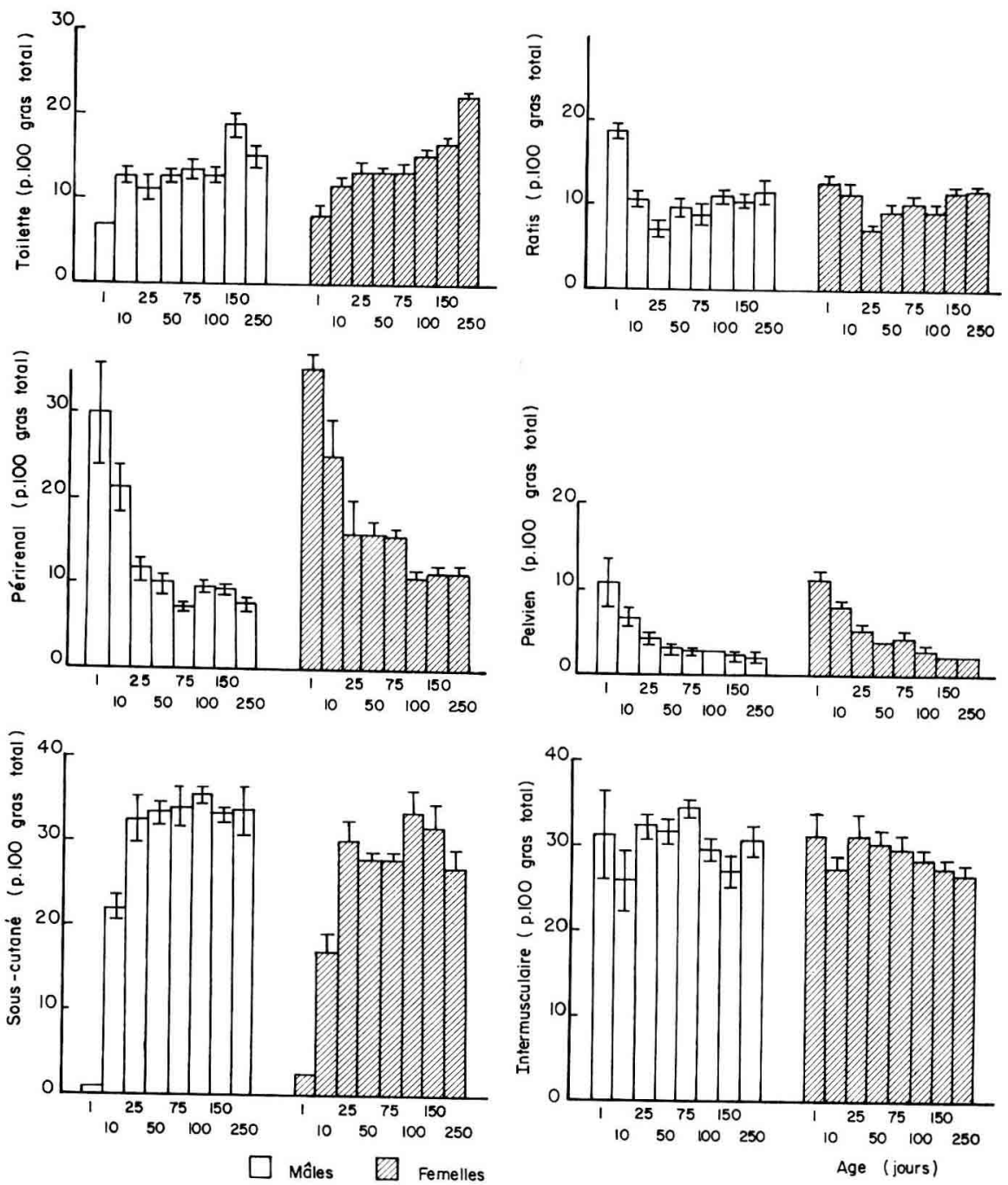

FIG. 2. - Evolution en fonction de l'âge des différents dépôts adipeux (moyenne \pm erreur standard) en p. 100 du gras total pour des agneaux Mérinos d'Arles des deux sexes

- Les dépôts adipeux périrénaux et de la cavité pelvienne se caractérisent dans les deux sexes par une réduction constante de leur pourcentage par rapport au gras total depuis la naissance jusqu'à la fin de la période étudiée. Cette réduction est surtout marquée entre I et 25 jours et apparaît plus nettement pour le gras périrénal qui représente à la naissance un important pourcentage du gras total. En outre, les femelles ont toujours des pourcentages supérieurs à ceux des mâles. 
- Les dépôts sous-cutanés subissent une évolution inverse des précédents, caractérisée par un important accroissement dans les 25 premiers jours de la vie. Après cette période le taux est sensiblement constant. Les mâles ont des pourcentages de gras de couverture toujours supérieurs à ceux des femelles de ro à 250 jours.

- Les dépôts adipeux intermusculaires représentent dans les deux sexes et tout au long de la croissance 25 à 35 p. Ioo des dépôts totaux; leur évolution est assez irrégulière, mais 1'on note une tendance, bien marquée chez la femelle, à perdre de leur importance relative au-delà de 25 jours. Ici encore les pourcentages sont, pour la plupart des âges étudiés, supérieurs chez les mâles par rapport aux femelles.

TABLEAU I $a$

Valeurs moyennes et (coefficients de variation $\left({ }^{1}\right)$ ) des différents dépôts adipeux d'agneaux Mérinos d'Arles mâles au cours de la croissance

\begin{tabular}{|c|c|c|c|c|c|c|c|c|}
\hline Age (j) & 0 & 10 & 25 & 50 & 75 & 100 & 150 & 250 \\
\hline Effectif & 2 & 4 & 6 & 10 & 7 & 13 & 6 & 6 \\
\hline $\begin{array}{c}\text { Poids vif vide } \\
(\mathrm{kg})\end{array}$ & $\begin{array}{l}3,92 \\
(2,2)\end{array}$ & $\begin{array}{l}6,14 \\
(8,9)\end{array}$ & $\begin{array}{l}8,93 \\
(5,2)\end{array}$ & $\begin{array}{l}13,28 \\
(15,9)\end{array}$ & $\begin{array}{l}14,29 \\
(29,6)\end{array}$ & $\begin{array}{r}20,30 \\
(12,7)\end{array}$ & $\begin{array}{l}23,81 \\
(4,5)\end{array}$ & $\begin{array}{l}33,27 \\
(9,9)\end{array}$ \\
\hline Gras total (g) & $\begin{array}{r}61,6 \\
(2,2)\end{array}$ & $\begin{array}{l}287,7 \\
(29,6)\end{array}$ & $\begin{array}{l}776,5 \\
(17,6)\end{array}$ & $\begin{array}{l}1329 \\
(37,4)\end{array}$ & $\begin{array}{l}1957 \\
(53,2)\end{array}$ & $\begin{array}{l}2049 \\
(41,3)\end{array}$ & $\begin{array}{l}3199 \\
(17,4)\end{array}$ & $\begin{array}{l}4207 \\
(11,8)\end{array}$ \\
\hline Toilette (g) & $\begin{array}{c}4,4 \\
(3,2)\end{array}$ & $\begin{array}{c}36,0 \\
(36,9)\end{array}$ & $\begin{array}{c}84,4 \\
(2 ', 7)\end{array}$ & $\begin{array}{l}164,9 \\
(41,6)\end{array}$ & $\begin{array}{r}257,6 \\
(68,6)\end{array}$ & $\begin{array}{l}261,4 \\
(43,1)\end{array}$ & $\begin{array}{l}587,7 \\
(26,9)\end{array}$ & $\begin{array}{l}623,5 \\
(24,3)\end{array}$ \\
\hline Ratis (g) & $\begin{array}{l}11,4 \\
(6,2)\end{array}$ & $\begin{array}{c}30,1 \\
(24,8)\end{array}$ & $\begin{array}{c}54,5 \\
(22,7)\end{array}$ & $\begin{array}{l}128,1 \\
(31,2)\end{array}$ & $\begin{array}{l}171,6 \\
(28,1)\end{array}$ & $\begin{array}{l}223,5 \\
(41,2)\end{array}$ & $\begin{array}{l}328,7 \\
(23,1)\end{array}$ & $\begin{array}{l}478,3 \\
(39,2)\end{array}$ \\
\hline $\begin{array}{c}\text { Gras périrénal } \\
(\mathrm{g})\end{array}$ & $\begin{array}{c}18,5 \\
(27,2)\end{array}$ & $\begin{array}{c}61,4 \\
(23,2)\end{array}$ & $\begin{array}{c}91,1 \\
(36,9)\end{array}$ & $\begin{array}{l}133,3 \\
(55,2)\end{array}$ & $\begin{array}{l}139,8 \\
(55,2)\end{array}$ & $\begin{array}{l}193,7 \\
(52,6)\end{array}$ & $\begin{array}{l}299,0 \\
(17,1)\end{array}$ & $\begin{array}{l}318,3 \\
(34,0)\end{array}$ \\
\hline Gras pelvien $(\mathrm{g})$ & $\begin{array}{c}6,7 \\
(20,2)\end{array}$ & $\begin{array}{c}18,7 \\
(18,2)\end{array}$ & $\begin{array}{c}32,1 \\
(34,8)\end{array}$ & $\begin{array}{c}39,4 \\
(44,0)\end{array}$ & $\begin{array}{c}52,5 \\
(25,2)\end{array}$ & $\begin{array}{c}57,0 \\
(39,3)\end{array}$ & $\begin{array}{c}70,5 \\
(33,1)\end{array}$ & $\begin{array}{c}85,9 \\
(31,0)\end{array}$ \\
\hline $\begin{array}{c}\text { Gras sous-cutané } \\
(\mathrm{g})\end{array}$ & 0,8 & $\begin{array}{c}63,7 \\
(30,5)\end{array}$ & $\begin{array}{l}251,8 \\
(36,9)\end{array}$ & $\begin{array}{l}442,1 \\
(41,9)\end{array}$ & $\begin{array}{r}656,9 \\
(57,6)\end{array}$ & $\begin{array}{l}759,8 \\
(44,6)\end{array}$ & $\begin{array}{l}1051 \\
(23,2)\end{array}$ & $\begin{array}{l}1409 \\
(27,4)\end{array}$ \\
\hline $\begin{array}{l}\text { Gras intermuscu- } \\
\text { laire }(\mathrm{g})\end{array}$ & $\begin{array}{c}19,4 \\
(25,2)\end{array}$ & $\begin{array}{c}74,8 \\
(52,1)\end{array}$ & $\begin{array}{l}251,9 \\
(17,4)\end{array}$ & $\begin{array}{l}420,8 \\
(45,6)\end{array}$ & $\begin{array}{l}676,9 \\
(56,6)\end{array}$ & $\begin{array}{l}627,7 \\
(1+2,6)\end{array}$ & $\begin{array}{l}861,0 \\
(18,1)\end{array}$ & $\begin{array}{l}1292 \\
(15,5)\end{array}$ \\
\hline
\end{tabular}

( $)$ Coefficient de variation $=\frac{\text { Écart-type }}{\text { Moyenne }} \times 100$.

- Un point mérite d'être souligné à propos de tous les résultats exposés ci-dessus, il concerne la variabilité des paramètres étudiés. Le tableau I réservé à cette étude met en évidence :

- Pour le poids vif vide une augmentation du coefficient de variation jusqu'à 75 jours pour les mâles, roo jours pour les femelles, puis une diminution régulière jusqu'à 250 jours. 
- Pour le gras total on note une évolution analogue mais le coefficient de variation est toujours beaucoup plus important qu'il ne l'était dans le cas du poids vif vide ; il atteint jusqu'à 53,2 p. Ioo à 75 jours chez les mâles et 45,9 p. I00 à I0o jours chez les femelles.

- Pour certains dépôts étudiés le phénomène est encore plus marqué ; les gras de toilette, périrénaux et sous-cutanés paraissent les plus variables.

- Enfin la variabilité semble d'une manière générale être moindre chez les femelles que chez les mâles.

\section{TABLEAU I $b$}

Valeurs moyennes et (coefficients de variation $\left({ }^{1}\right)$ ) des différents dépôts adipeux d'agneaux Mérinos d'Arles Femelles au cours de la croissance

\begin{tabular}{|c|c|c|c|c|c|c|c|c|}
\hline Age (j) & 0 & 10 & 25 & 50 & 75 & 100 & 150 & 250 \\
\hline Effectif & 2 & $t_{t}^{\prime}$ & 4 & 8 & 5 & 10 & 6 & 6 \\
\hline $\begin{array}{l}\text { Poids vif vide } \\
(\mathrm{kg})\end{array}$ & $\begin{array}{r}3,93 \\
(3,1)\end{array}$ & $\begin{array}{l}5,82 \\
(3,8)\end{array}$ & $\begin{array}{l}8,93 \\
(2,3)\end{array}$ & $\begin{array}{l}11,69 \\
(6,5)\end{array}$ & $\begin{array}{l}15,50 \\
(7,3)\end{array}$ & $\begin{array}{l}18,61 \\
(15,6)\end{array}$ & $\begin{array}{l}20,30 \\
(8,8)\end{array}$ & $\begin{array}{l}26,62 \\
(5,37)\end{array}$ \\
\hline Gras total (g) & $\begin{array}{r}82,4 \\
(2,5)\end{array}$ & $\begin{array}{l}259,0 \\
(6,9)\end{array}$ & $\begin{array}{l}829,8 \\
(8,5)\end{array}$ & $\begin{array}{l}1456 \\
(21,6)\end{array}$ & $\begin{array}{l}1809 \\
(20,7)\end{array}$ & $\begin{array}{l}2607 \\
(45,9)\end{array}$ & $\begin{array}{l}3356 \\
(17,5)\end{array}$ & $\begin{array}{l}4771 \\
(25,4)\end{array}$ \\
\hline Toilette (g) & $\begin{array}{c}6,6 \\
(21,4)\end{array}$ & $\begin{array}{c}29,7 \\
(41,7)\end{array}$ & $\begin{array}{l}109,0 \\
(23,2)\end{array}$ & $\begin{array}{l}191,6 \\
(26,9)\end{array}$ & $\begin{array}{l}236,3 \\
(25,7)\end{array}$ & $\begin{array}{l}390,5 \\
(48,9)\end{array}$ & $\begin{array}{l}557,0 \\
(23,0)\end{array}$ & $\begin{array}{l}1052 \\
(24,8)\end{array}$ \\
\hline Ratis (g) & $\begin{array}{l}10,4 \\
(4,7)\end{array}$ & $\begin{array}{c}28,3 \\
(23,4)\end{array}$ & $\begin{array}{l}58,2 \\
(7,7)\end{array}$ & $\begin{array}{l}129,8 \\
(33,3)\end{array}$ & $\begin{array}{l}178,0 \\
(18,2)\end{array}$ & $\begin{array}{l}235,0 \\
(44,8)\end{array}$ & $\begin{array}{l}381,8 \\
(25,6)\end{array}$ & $\begin{array}{l}553,6 \\
(27,2)\end{array}$ \\
\hline $\begin{array}{l}\text { Gras périrénal } \\
(\mathrm{g})\end{array}$ & $\begin{array}{r}29,0 \\
(4,4)\end{array}$ & $\begin{array}{c}63,9 \\
(27,2)\end{array}$ & $\begin{array}{l}131,4 \\
(40,2)\end{array}$ & $\begin{array}{l}229,8 \\
(37,6)\end{array}$ & $\begin{array}{l}282,4 \\
(32,3)\end{array}$ & $\begin{array}{l}275,8 \\
(47,5)\end{array}$ & $\begin{array}{r}374,0 \\
(32,8)\end{array}$ & $\begin{array}{l}527,5 \\
(44,2)\end{array}$ \\
\hline Gras pelvien (g) & $\begin{array}{c}9,2 \\
(7,6)\end{array}$ & $\begin{array}{c}20,7 \\
(19,3)\end{array}$ & $\begin{array}{c}40,3 \\
(21,7)\end{array}$ & $\begin{array}{c}54,7 \\
(19,6)\end{array}$ & $\begin{array}{c}7 t, 0 \\
(22,4)\end{array}$ & $\begin{array}{l}67,8 \\
(33)\end{array}$ & $\begin{array}{c}71,9 \\
(28,7)\end{array}$ & $\begin{array}{l}102,5 \\
(31,0)\end{array}$ \\
\hline $\begin{array}{c}\text { Gras sous-cutané } \\
(\mathrm{g})\end{array}$ & $\begin{array}{c}2,0 \\
(3,4)\end{array}$ & $\begin{array}{c}44,2 \\
(54,5)\end{array}$ & $\begin{array}{l}248,8 \\
(22,9)\end{array}$ & $\begin{array}{l}404,3 \\
(27,7)\end{array}$ & $\begin{array}{r}505,0 \\
(25,7)\end{array}$ & $\begin{array}{l}864,6 \\
(67,8)\end{array}$ & $\begin{array}{l}1057 \\
(20,5)\end{array}$ & $\begin{array}{r}1272 \\
(29,7)\end{array}$ \\
\hline $\begin{array}{l}\text { Gras inter- } \\
\text { musculaire (g) }\end{array}$ & $\begin{array}{r}25,5 \\
(9,1)\end{array}$ & $\begin{array}{c}70,6 \\
(34,8)\end{array}$ & $\begin{array}{l}260,8 \\
(9,1)\end{array}$ & $\begin{array}{l}441,0 \\
(18,2)\end{array}$ & $\begin{array}{l}531,9 \\
(16,6)\end{array}$ & $\begin{array}{l}725,4 \\
(44,2)\end{array}$ & $\begin{array}{l}911,6 \\
(23,5)\end{array}$ & $\begin{array}{l}1264 \\
(19,6)\end{array}$ \\
\hline
\end{tabular}

(1) Coefficient de variation $=\frac{\text { Écart-type }}{\text { Moyenne }} \times 100$.

\section{2. - Influence de l'hypophysectomie et de traitements chroniques à la $B G H$}

Comparativement aux animaux normaux, les agneaux hypophysectomisés traités ou non à la $\mathrm{BGH}$ présentent, par rapport aux animaux normaux abattus au même âge qu'eux, des différences plus ou moins marquées dans l'état d'adiposité des carcasses. 
Les comparaisons ont été faites à Ioo, I 50 et 250 jours, les opérations ayant eu lieu respectivement à 25,50 et Ioo jours.

Les résultats (fig. 3) mettent en évidence les points suivants :

- à une exception près (mâles âgés de roo jours) l'hypophysectomie induit une surcharge en tissus adipeux des carcasses ; celle-ci est encore plus accusée si la comparaison est faite avec les sujets pseudoopérés ayant comme les hypophysectomisés subi un choc opératoire;

- le traitement chronique à la BGH des hypophysectomisés provoque au contraire, un appauvrissement très net de la teneur en graisse des carcasses par comparaison aux sujets normaux et à une exception près (femelles âgées de I $_{5}$ o jours) aux pseudoopérés.

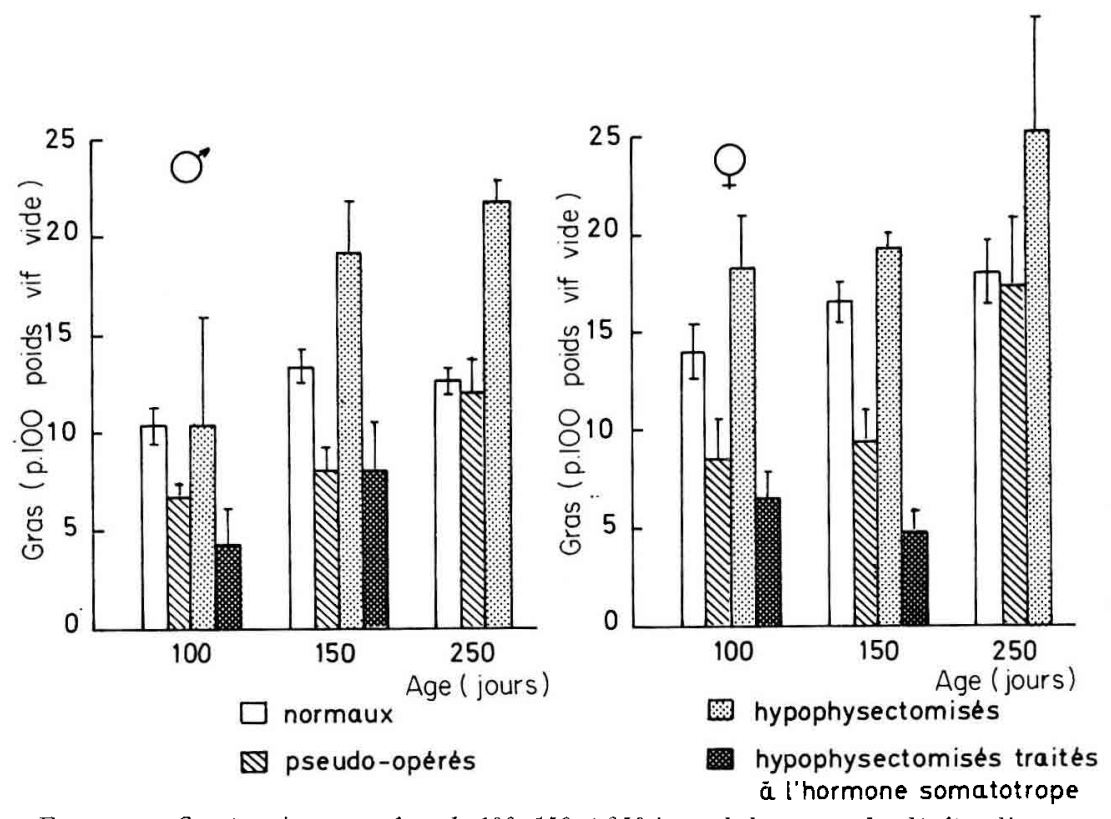

FIG. 3.-- Comparaison aux âges de 100, 150 et 250 jours de la somme des dépôts adipeux

(moyenne \pm erreur standard) en $p .100$ du poids vif vide pour des agneaux Mérinos d'Arles normaux, pseudoopérés, hypophysectomisés et hypophysectomisés traités chroniquement à l'hormone somatotrope

Au tableau 2 nous donnons en p. Ioo du gras total la répartition des dépôts adipeux étudiés pour les animaux des différents lots expérimentaux.

- Les gras mésentériques, de toilette et de ratis des agneaux hypophysectomisés sont toujours plus faibles surtout chez les femelles que chez les témoins intacts ou, à de rares exceptions près, que chez les pseudoopérés.

- Le développement relatif du gras périrénal est par contre toujours nettement accru par l'hypophysectomie dans les deux sexes.

- Les parts du gras total attribuables aux gras pelvien, sous-cutané et intermusculaire ne subissent que peu de changement par rapport à ce que l'on observe chez les agneaux normaux. On peut signaler cependant une proportion légèrement plus forte en gras sous-cutané chez les agneaux mâles hypophysectomisés de I5o jours. 


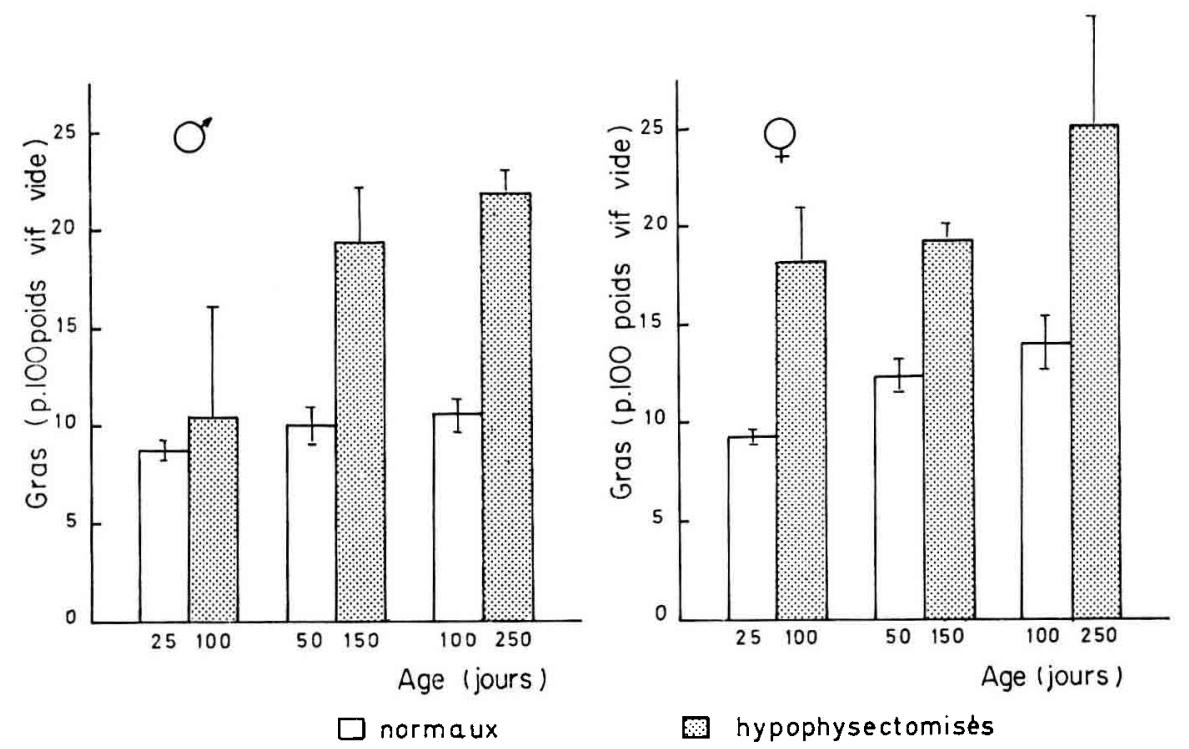

FIG. 4. - comparaison (moyenne \pm erreur standard) en $p$. 100 du poids vif vide, du gras total d'agneaux hypophysectomisés abattus à 100, 150 et 250 jours et d'agneaux normaux abattus aux âges de 25,50 et 100 jours, ces âges correspondant aux stades respectifs d'hypophysectomie des opérés

\section{TABLEAU 3}

Comparaison, en p. 100 du gras total, des différents dépóts adipeux d'agneaux hypophysectomisés abattus à 100, 150 et 250 jours et d'agneaux normaux abattus aux âges de 25, 50 et 100 jours, ces âges correspondant aux stades respectifs d'hypophysectomie des opérés

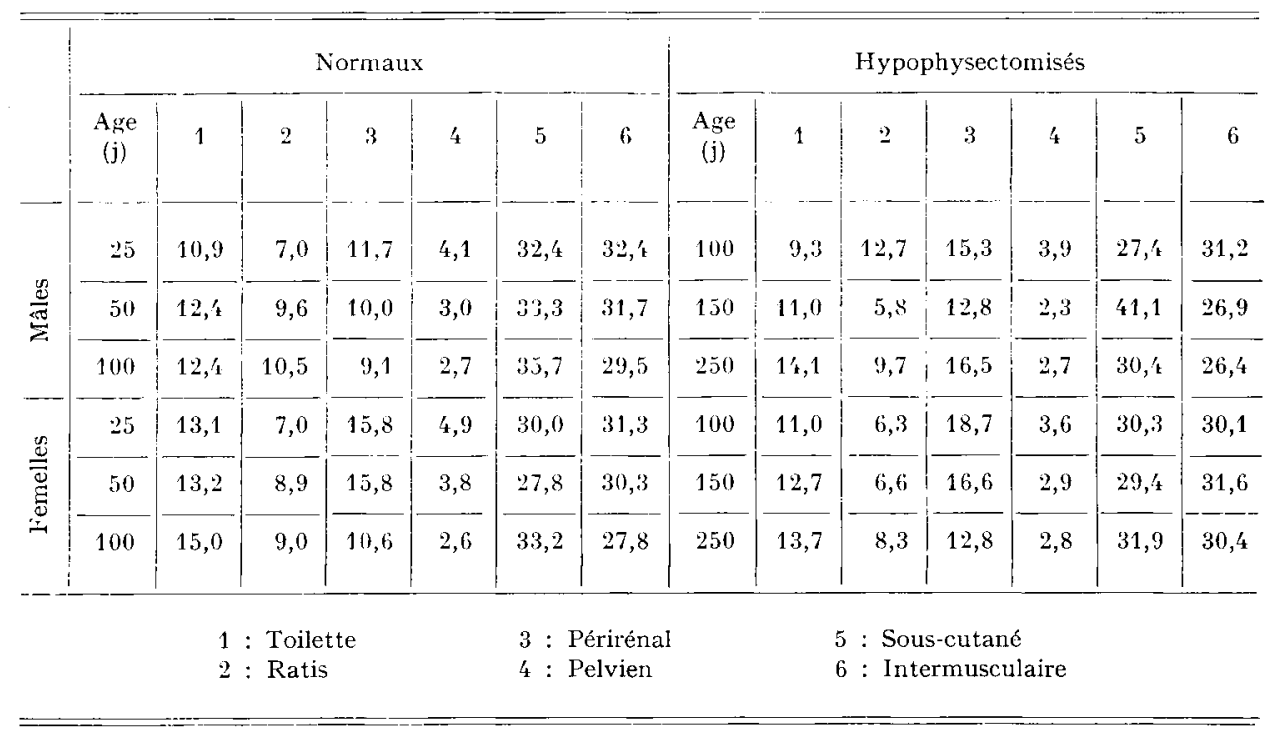


- Les animaux pseudoopérés ne présentent pas de variations nettes de l'impor tance des différents tissus adipeux étudiés par rapport aux animaux normaux.

Le traitement chronique à la BGH des agneaux hypophysectomisés rétablit une répartition des tissus adipeux sensiblement voisine de celle des témoins normaux avec dans certains cas une tendance à un développement relatif légèrement plus important du gras mésentérique et légèrement moindre du gras intermusculaire.

L'important développement des dépôts périrénaux observé chez les agneaux hypophysectomisés étant une caractéristique des stades de développement précoces, il nous a semblé intéressant de comparer les individus privés d'hypophyse avec les agneaux normaux abattus à l'âge même où sont pratiquées les opérations soit 25 , 50 et roo jours.

Dans tous les cas, la masse adipeuse rapportée au poids vif vide (fig. 4) est nettement accrue chez les hypophysectomisés, l'accroissement étant d'autant plus grand que la survie est plus longue et que l'animal est opéré plus tardivement.

Par contre (tabl. 3) la répartition des différents types de gras par rapport au gras total reste sensiblement la même qu'au moment de l'opération, seul le gras périrénal présente une légère supériorité chez les agneaux hypophysectomisés notamment chez les mâles.

\section{DISCUSSION}

Les résultats concernant l'évolution des proportions des dépôts adipeux totaux par rapport au poids vif vide confirment en grande partie ceux de BÉNÉVENT (I97I) obtenus sur un cheptel génétiquement semblable mais élevé dans des conditions de milieu différentes selon un rythme de croissance prédéterminé ; ils les complètent pour la période située au-delà de 160 jours.

Le point le plus remarquable est l'existence d'une véritable pause dans l'accroissement relatif des dépôts adipeux pour les deux sexes.

Nous n'avons pas noté comme BÉNÉVENT de réduction de l'importance moyenne des dépôts sous-cutanés. Ėn ce qui concerne les différents types de gras, on peut les classer en dépôts de développement :

- précoce (gras périrénal, pelvien) ;

- intermédiaire (gras intermusculaire et de ratis);

- tardif (gras de couverture et de toilette).

La nature des dépôts adipeux peut en partie expliquer cet ordre de précocité dans le développement, notamment pour le tissu périrénal qui possède à la naissance de l'agneau des caractéristiques de tissu brun ce qui pourrait justifier qu'il regresse rapidement laissant place au tissu adipeux blanc.

L'ordre de précocité rencontré chez l'Agneau est en apparence différent de celui rencontré chez le Lapin (PRUD'hon, VÉzINHET et CANTIER, r970) ou le Rat (REED, I930). Dans ces espèces où les jeunes sont dépourvus de pilosité à la naissance, le tissu sous cutané est d'un développement très précoce et intenșe afin semble-t-il d'assurer avant l'apparition des poils une protection thermique suffisante, leur 
importance régresse ensuite. Chez l'Agneau, le développement est également rapide dans les jours qui suivent la naissance mais plus progressif, prolongé et ne marque pas de régression.

En outre Boccard et DUPLAN (I96I), comparant des carcasses d'agneaux Ile-de-France de $17,5 \mathrm{~kg}$ obtenues à partir de croissance moyenne $(250 \mathrm{~g} / \mathrm{j})$ et rapide $(350 \mathrm{~g} / \mathrm{j})$ avaient constaté qu'à ce stade les dépôts sous-cutanés avaient été davantage influencés par la vitesse de croissance que les dépôts intermusculaires. Ce qui va dans le même sens que les résultats que nous obtenons à I5o jours et au-delà dans la mesure où l'on admet qu'une vitesse de croissance supérieure correspond à un développement physiologique plus rapide.

Pour ce qui concerne l'interprétation physiologique à donner à nos résultats, INGLE et al. (I972) ont signalé que chez 1'Agneau, la synthèse des acides gras de novo et in situ semble en relation avec le degré de précocité du dépôt gras. C'est ainsi que chez le jeune, la synthèse des lipides a surtout lieu dans le dépôt périrénal alors que chez l'adulte, elle semble prédominer au niveau du gras de couverture. L'évolution observée ici pour les gras internes (périrénal, pelvien et du ratis) va dans le sens d'une diminution de l'importance relative de ces gras, tandis que dans le même temps les gras sous-cutanés augmentent leur pourcentage par rapport au gras total. Ces résultats pourraient alimenter l'hypothèse d'une prise de relais de la fonction lipogénique des gras internes par les gras sous-cutanés. Une exception, le gras de toilette, pour lequel simultanément au développement du rumen on observe un accroissement de l'importance relative.

Nos résultats ont par ailleurs mis en évidence l'extrême variabilité, à âge égal, des différents dépôts adipeux; une comparaison des mêmes données à poids vif vide égal diminue un peu, sans pour autant la supprimer, la variabilité des dépôts intermusculaires ou de ratis qui étaient déjà les moins sujets à variation.

Ce phénomène a déjà été souligné par LEGRAs (I970) pour des carcasses de femelles ovines d'âges différents et par Dumont et al. (I96I) chez les bovins; il implique que l'on constitue des lots expérimentaux d'effectif important, chaque fois que l'on désire étudier le développement des dépôts adipeux des carcasses et à l'intérieur de ces lots, que l'on compare entre eux des sujets ayant eu les mêmes caractéristiques de croissance. Il reste que, pour le tissu gras, éliminer la variabilité est une chose difficile, ce qui tient au fait que l'importance du tissu adipeux varie rapidement en réponse à des facteurs d'origine interne et externe (facteurs endocriniens, nutritionnels, climatiques, etc.).

En ce qui concerne les effets de l'hypophysectomie sur le développement des tissus adipeux, les résultats de VézinHE'T (I973) sont complétés et approfondis. Il apparaît nettement que chez les animaux opérés la croissance musculaire et osseuse est arrêtée mais la croissance du tissu gras se déroule normalement, les dépôts formés ayant la même répartition qu'au moment de l'opération, avec une prédominance cependant des dépôts périrénaux.

Ce fait pourrait étayer l'hypothèse que l'importance respective des différents dépôts adipeux et de leurs variations au cours de la croissance dépend essentiellement des modifications de longueur, de surface et de volume des sites de dépôt, toutefois on explique mal alors l'importance particulière du gras périrénal chez les hypophysectomisés.

Enfin, les effets d'un traitement à la $\mathrm{BGH}$, qui provoque l'amaigrissement des 
carcasses, confirme le rôle lipolytique bien connu de cette hormone. Ce rôle semble s'exercer de façon uniforme sur les différents sites de dépôts du tissu gras, comme en témoigne la répartition inchangée des graisses corporelles.

Reçu pour publication en juillet 1973.

\section{REMERCIEMENTS}

Ce travail a été en partie réalisé grâce à l'aide d'un contrat de la Délégation Générale de la Recherche Scientifique et Technique no 7 I 7 3rag et d'une Action Thématique Programmée de l'Institut national de la Recherche agronomique.

\section{SUMMARY}

\section{EVOLUTION OF DIFFERENT TYPES OF FAT DEPOSITS AFTER BIRTH IN NORMAI, OR HYPOPHYSECTOMIZED MERINOS D'ARLES LAMBS}

Evolution of different types of fat deposits during postnatal life has been studied in 99 normal Mérinos d'Avles lambs of the two sexes, and in 45 lambs undergoing hypophysectomy or a sham operation. Some hypophysectomized lambs received a chronic treatment with bovine somatotropic hormone $(\mathrm{BGH})$. In relation to empty weight, fat deposits take on relatively increasing importance during postnatal life in the period studied. In different types of deposits, there is an almost constant proportion of large epiploon fat (IO-12 P. IOo of total fat) and intermuscular deposits (25-30 p. Ioo of total fat). Perirenal and pelvic deposits diminish while sub-cutaneous and small epiploon deposits increase. The latter evolve analogously to total deposits. Data obtained vary greatly, especially for perirenal and sub-cutaneous deposits.

Hypophysectomy causes increased carcass fattening. Distribution of different fat deposits of hypophysectomized lambs differs markedly from that of normal lambs of the same age, and, except for perirenal tissue which is more developed in operated lambs, distribution differs little from that observed in lambs slaughtered at the age at which hypophysectomy was performed.

Chronic BGH treatment of hypophysectomized lambs causes carcass fat loss, thus confirming the lipolytic role of this hormone.

\section{RÉFÉRENCES BIBLIOGRAPHIQUES}

BÉNÉVEnt M., r968. Guide pour la dissection et l'identification des principaux muscles de la carcasse chez le Mouton. Ann. Biol. anim. Biochim. Biophys., 8, ro7-139.

BÉn f́vent M., I97I. Croissance relative pondérale postnatale dans les deux sexes des principaux tissus et organes de l'Agneau Mérinos d'Arles. Ann. Biol. anim. Bioch. Biophys., 11, 5-39.

Boccard R., Duplan J. M., I96r. Étude de la production de viande chez les ovins. III. Note sur l'influence de la vitesse de croissance sur la composition corporelle des agneaux. Ann. Zootech. 10, 31 -38.

Dumont B. L., I971. La viande d'agneau de boucherie. Qualités des ovins de boucherie. Pâtre, 183, 3-Iz.

Dumont B. L., Le Guelte P., ARnoux J., I96I. Étude biométrique des bovins de boucherie. I. Variabilité de la composition anatomique de la carcasse des bovins charolais. Ann. Zootech., 10, I49-I54.

Flamant J. C., Boccard R., I966. Estimation de la qualité de la carcasse des agneaux de boucherie. Ann. Zootech., 15, 89-1 13 .

Henky Y., 1972. Facteurs de variation de la composition des dépôts adipeux chez le Porc. Rev. Française des Corps Gras, 22, 367-376.

Ingle D. L., Bauman D. E., Garrigus U. S., 1972. Lipogenesis in the ruminant: in vivo site of fatty acid synthesis in sheep. J. Nutr., 102, 617-624. 
Khachadurian A. K., Adrouni B., Yacoubian H., ig66. Metabolism of adipose tissue in the fat tail of the sheep in vivo. J. Lipid. Res., 7, 427-436.

Legras P., 197o. Évolution avec l'âge des qualités de carcasse et de viande des femelles ovines. Pâtre, 179, I 5-I7.

Martin A. H., Freeden H. T., Weiss G. M., Carson R. B., I972. Distribution and composition of carcass fat. J. Anim. Sci, 35, 534-54I.

McClelland T. H., Russel A. J. F., 1972. The distribution of body fat in scottish blackface and finnish landrace lambs. Anim. Prod, 15, 301-306.

Prud'hon M., Vézinhet A., Cantier J., ig7o. Croissance, qualités bouchères et coût de production des lapins de chair. Bull. tech. Inf. Ingrs Servs agric., 248, I-IO.

Prud'hon M., Reyne Y., Garambois X., I972. Estimation de la composition corporelle d'agneaux Mérinos d'Arles abattus à des stades de croissance compris entre la naissance et un an. Ann. Zootech., 21, 299-309.

Reed L. L., Yamaguchi F., Anderson W. E., Mendel L. B., i93o. Factors infuencing the distribution and character of adipose tissue in the rat. J. biol. Chem., 87, 147.

Richmond R. J., Berg R. T., r971. Fat distribution in swine as influenced by liveweight, breed, sex and ration. Can. J. Anim. Sci., 51, 523-531.

Russel A. J. F., Doney J. M., Gunn R. G., I97I. The distribution of chemical fat in the bodies of scottish black face ewes. Anim. Prod., 13, 503-509.

Shafrir E., Wertheimer E., ig65. In : Renold A. E., Cahill G. F. Adipose tissue. Handbook of physiology. Amer. Physiol. Soc. Washington. 803 p.

VÉzINHET A., 1973. Influence de l'hypophysectomie et de traitements à l'hormone somatotrope bovine sur la croissance relative de l'Agneau. Ann. Biol. anim. Bioch. Biophys 13, 5 I-73. 